Echeverría Samanes, B. (2016). Transferencia del Sistema de FP Dual a España. Revista de Investigación Educativā, 34(2), 295-314.

DOI: http://dx.doi.org/10.6018/rie.34.2.249341

\title{
Transferencia del sistema de FP Dual a España
}

\section{Dual Vocational Training System Transferral to Spain}

\author{
Benito Echeverría Samanes \\ Universidad de Barcelona
}

\begin{abstract}
Resumen
La presión ejercida por diversos organismos comunitarios a favor de la formación profesional dual es patente en España, donde está regulado su desarrollo desde finales de 2012. Transcurrido un trienio, se analiza el proceso de implantación, a través de una investigación-acción, realizada en seis estados de la UE y financiada por el CEDEFOP. El artículo sintetiza las valoraciones de más de 40 expertos españoles, que alertan sobre las dificultades existentes, reflejan las deficiencias observadas y aportan pautas de actuación para un correcto establecimiento de este sistema formativo en todas las CCAAs del estado español.

Palabras clave: aprendizajes profesionales; formación profesional dual; orientación profesional coordinada; investigación-acción.
\end{abstract}

\begin{abstract}
The pressure of implementing the Dual Vocational Training System by several European institutions was apparent in Spain, where this development has been regulated since the end of 2012. After three years, the implantation process was analyzed with action research methodology, which was done by six different states of the EU and it was financed by CEDEFOP. This paper summarizes the assessments of over forty Spanish experts about this process. The real difficulties and deficiencies were observed and reflected by these experts who contributed the proper steps for a correct establishment of this training system in all Spanish regions.

Keywords: professional learning; dual vocational training system; coordinate career guidance; action research.
\end{abstract}

Correspondencia: Benito Echeverría Samanes, Dpto. MIDE. Universidad de Barcelona. Edificio de Levante, Pso. Valle Hebrón 171 - 08035 Barcelona, (España). E-mail: becheverria9@gmail.com 


\section{Apremio institucional}

El interés por implantar en España el sistema de formación profesional dual (en adelante FPD) - paradigmático de Alemania, Austria y Suiza - ha ido apareciendo para luego eclipsarse, a lo largo de toda mi actividad profesional; Primero tras la crisis del petróleo de los años $70^{\prime}$, luego en la de los años 90' y ahora en plena crisis financiera del siglo XXI. Ha sido durante ésta última, cuando varios organismos internacionales se han pronunciado claramente a favor de su instauración.

Desde el inicio de esta década, la OCDE (2010) mostró su "reconocimiento a escala mundial" del sistema dual en su informe sobre Alemania (Hoeckel \& Schwartz, 2010, p. 12). Poco después, la Confederación Europea de Negocios (BUSINESSEUROPE, 2012) aconsejó implantar "sistemas de FP duales" en los 27 países de la UE dentro de un conjunto de medidas destinadas a mejorar programas de aprendizaje y prácticas profesionales, elaborados a petición de la Comisión Europea (CE). Esta última institución ya había reclamado en el Comunicado de Brujas (CE, 2010, p. 11) "ampliar al máximo la formación basada en el trabajo, incluido el aprendizaje en centros de trabajo" y desde su comunicado estratégico de "un nuevo concepto de educación" (CE, 2012a) viene propugnando que

el aprendizaje en el lugar de trabajo, por ejemplo con los modelos duales, debe ser un pilar central de los sistemas de educación y formación profesionales en toda Europa, con el objetivo de reducir el desempleo juvenil, facilitar la transición del aprendizaje al empleo y responder a las necesidades de competencias del mercado laboral (p. 8).

Con estos fines se creó la Alianza Europea de Aprendizaje (en Leipzig, 2013), respaldada por una novedosa declaración conjunta del Consejo (CE, 2013), Comisión y federaciones europeas de sindicatos y de empresarios comunitarios (EAA, 2013). A través de ella, autoridades públicas, empresas, sindicatos, cámaras de comercio, promotores de educación y formación profesional, representantes de la juventud, servicios de empleo y otros actores clave, tratan de: a) Apoyar a los sistemas educativos que pretenden introducir o reformar aspectos esenciales del aprendizaje basado en el trabajo; b) Promocionar estudios sobre este modelo de aprendizaje; c) Estimular las medidas promovidas por la Alianza con recursos del Fondo Social Europeo, Garantía Juvenil, Erasmus + (Echeverría, 2013a).

Especialmente significativa es la estrecha colaboración del gobierno y cámaras de comercio e industrias alemanas, interesados en difundir el sistema de FPD sobre todo en los países sureños de la UE y, últimamente, más allá de la Comunidad. El Ministerio Federal de Educación e Investigación explica claramente los motivos:

la exportación de servicios de formación y profesionalización... tiene un efecto de palanca para la industria alemana, ya que la exportación de bienes, por ejemplo, en la construcción de maquinaria o en la industria automovilística a menudo exige obligatoriamente la existencia de técnicos bien formados en el extranjero (BMBF, 2012, p. 74). 
Prueba de esta decidida apuesta por extender el sistema de FPD es el gran número existente de proyectos de cooperación germanos, promovidos a nivel político y potenciados por organizaciones como el Instituto Federal de Formación Profesional (BIBB), Agencia Cooperación Internacional (GIZ), etc. A ellas se han unido recientemente fundaciones como la del Grupo de empresas de medios de comunicación -Bertelsmann-, cuya actividad en nuestro país apuesta ahora "Por el empleo juvenil", mediante la formación y orientación profesional, tras dedicarse 20 años a fomentar el interés por la lectura entre niños y jóvenes y la mejora del sistema de bibliotecas públicas.

En muy poco tiempo, la Fundación Bertelsmann se ha convertido en la principal impulsora en nuestro país de la Alianza para la Formación Profesional Dual (AFPD, 2015), junto a la Fundación Princesa de Girona, la CEOE y las Cámaras de Comercio, pero sin participación de sindicatos. Su meta es "mejorar la empleabilidad de los jóvenes españoles al mismo tiempo que las empresas obtienen profesionales con una formación más acorde a sus necesidades". En concreto trata de: a) Extender el modelo de FPD, garantizando la calidad del aprendizaje en las empresas; b) Fomentar la colaboración y apoyo necesario para que las pequeñas y medianas empresas puedan sumarse a la FPD y ofertar plazas de aprendizaje; c) Conseguir que la experiencia de las empresas, centros e instituciones en la implantación de la FPD sirva para revisar y mejorar la regulación estatal y autonómica existente; d) Aumentar el prestigio social de la FP en general y fomentar que el modelo de aprendizaje de la FPD sea valorado y adoptado por la sociedad en general y por las empresas en particular.

Una de las iniciativas más reveladora de estas colaboraciones es el Centro Dual + para la Investigación de la FP Dual, desarrollado por Bankia y la Fundación alemana, para "generar conocimiento y crear espacios de análisis y concertación", mediante programas de investigación básica, desarrollo regional y de modelos, formatos y procesos.

Los primeros "tienen como meta... diseñar y comprobar teorías e hipótesis, para conseguir así una base para el conocimiento orientado a la aplicación” en los programas de desarrollo regional, hasta perfilar el modelo más adecuado para trabajar en cada uno de los territorios con sus empresas y centros de FP. Se pretende así canalizar todo este potencial hacia el desarrollo de sistemas, formatos y procesos concretados en nuevos modelos de aprendizaje, modernización metodológica y tecnológica de los centros, impulso a la innovación en Pymes, etc. (Bankia, 2015).

Es previsible que ante tantas entidades interesadas por la FPD y tan peculiares contribuciones a su implantación y desarrollo, más de un lector interrogue por los motivos que las guían, como a veces hemos escuchado.

\section{ADN del sistema}

Más difícil de explicar son las prisas de nuestros gobernantes por decretar un sistema inusual en España, sin haber cumplido su primer año de su legislatura (RD 1529/2012) e incluso antes de ver la luz la mayoría de comunicados, alianzas y proyectos comunitarios, antes referidos. Sin el más mínimo diagnóstico de necesidades y aún menos de posibilidades, el gobierno del Partido Popular optó por poner en funcionamiento un "conjunto de... acciones e iniciativas formativas, mixtas de empleo y formación, 
que tienen por objeto la cualificación profesional de los trabajadores en un régimen de alternancia de actividad laboral en una empresa con la actividad formativa recibida en el marco del sistema de formación profesional para el empleo o del sistema educativo" (Art. 2.1).

Desde nuestra óptica se ha vuelto a cometer el típico error de intentar transportar sistemas educativos en containers, sin comprender su esencia y adoptar las prácticas adecuadas, emanadas de procesos interpretativos sobre los factores claves de éxito y del contexto donde se pretende instaurar. Más aún, si se quiere transferir la FPD germánica con varios agentes implicados, que han de negociar y acordar contenidos y condiciones de la formación y del contrato, entre otros muchos aspectos.

- El Estado establece el marco legal mediante: a) La ley de la formación profesional (Berufsbildungsgesetz) que fija las condiciones de su desarrollo a nivel estatal. Regula aspectos como obligaciones de los aprendices y maestros, requisitos generales de las empresas interesadas en formar, reconocimiento de oficios de formación, remuneración de los aprendices, sistema de exámenes. Así, asegura unos estándares de calidad y promueve la aceptación estatal (e internacional) de la titulación; b) El marco legal de reglamentación de la formación (Ausbildungsordnung) encargada a la empresa. Es diferente para cada oficio e incluye denominación del mismo, reconocimiento estatal, duración de la formación, destrezas y conocimientos a enseñar y requerimientos de los exámenes. Además, establece un plan de formación, tanto a nivel temporal, como de contenidos; c) El plan de estudios (Rahmenlehrplan) donde se describen objetivos y contenidos de las clases, diferentes para cada oficio. Dada la diversidad de experiencias de los aprendices, el plan de estudios es general y puede variar de un Estado Federal (Länd) a otro.

- Los sindicatos colaboran junto a la patronal en la configuración de los programas formativos. Además, juegan un papel esencial en la negociación de sueldos, vacaciones, duración del tiempo de prueba o posibilidad de continuar en la empresa tras la formación. Controlan por tanto todo el proceso y están a disposición de los aprendices ante cualquier conflicto o para resolver situaciones problemáticas. Estos pueden afiliarse a los sindicatos y obtener así protección legal y derechos sociales, deduciéndose del salario la cuota de afiliación.

- Las empresas ofrecen los puestos de formación, pagan los sueldos, se responsabilizan de los contenidos prácticos de la formación y ejercen una función tutorial, con el fin de que los aprendices adquieran las competencias necesarias y las demuestren en las pruebas finales. Para poder ofertar plazas de formación, han de cumplir ciertos requisitos como disponer de personal suficiente y con la cualificación necesaria para ejercer de formadores, lo cual explica que aproximadamente dos tercios de los aprendices alemanes se formen en grandes y medianas empresas.

- Las cámaras juegan un papel fundamental en la organización y coordinación de las empresas, muy esencial cuando éstas son PYMES. Deciden cuáles pueden colaborar en la FPD y los formadores pertinentes. Además, aconsejan, asisten y controlan la formación en las mismas, registran los contratos de formación y realizan los exámenes. 
La financiación del sistema corre principalmente a cargo de las empresas, que sufragan unos 2/3 del coste total. El Estado y los Länders asumen 1/4 parte y el resto corre a cargo de la Agencia de Empleo Alemana.

Las empresas asumen los gastos derivados de los 3 o 4 días semanales que el aprendiz dedica a formarse en sus sedes. Incluyen costes de personal -tanto del aprendiz como del encargado de su formación-, del puesto de trabajo, del taller o lugar donde se realiza la formación y las clases dentro de la empresa, junto a otros indirectos como las tasas de las Cámaras. A cambio, las empresas pueden adecuar los contenidos de la formación a sus necesidades y expectativas, además de conocer bien a aquellos aprendices que pueden interesarle contratar al final de su formación.

Por su parte, el Estado dedica aproximadamente el 7\% del presupuesto de educación a este sistema formativo, para pagar costes de personal, espacios y material de enseñanza de las escuelas de formación.

En síntesis. Se trata de un sistema que requiere esfuerzos compartidos no solo de entidades, sino también de personas; Los aprendices se ven obligados a compaginar horarios de estudio y laborales. El profesorado ha de flexibilizar el desarrollo de las materias. Y, los tutores de empresas han de proporcionar espacios de aprendizaje a los aprendices, guiarlos y evaluarlos, mientras desarrollan las funciones de su puesto de trabajo.

\section{Versión española}

Es evidente que la configuración del sistema alemán dista mucho del que se pretende desarrollar en España, empezando por el substrato del tejido empresarial. Nuestras medianas y grandes empresas no pasan del $2 \%$ del total y nuestras microempresas aportan un $40 \%$ del empleo. Por contra, la mayor aportación al empleo de Alemania procede de las grandes compañías (37,2\%) (Tabla 1) y cuenta con centros de formación supra-empresariales, inexistentes en nuestro país, donde se instruyen los aprendices de las pequeñas empresas.

Tabla 1

Tejido empresarial español y alemán.

\begin{tabular}{lcccc}
\hline \multicolumn{2}{c}{ No de empresas (\% sobre el total) } & \multicolumn{2}{c}{$\begin{array}{c}\text { Aportación al empleo por tamaño } \\
\text { de empresa }\end{array}$} \\
\hline Trabajadores & ESPAÑ & ALEMANIA & ESPAÑ & ALEMANIA \\
\hline Micro (0-9) & $93,8 \%$ & $83,2 \%$ & $40,1 \%$ & $19,3 \%$ \\
Pequeñas (10-49) & $5,4 \%$ & $13,7 \%$ & $21,2 \%$ & $23,1 \%$ \\
Medianas (50-249) & $0,7 \%$ & $2,6 \%$ & $14,1 \%$ & $20,5 \%$ \\
Grandes (+ 250) & $0,1 \%$ & $0,5 \%$ & $24,6 \%$ & $37,2 \%$ \\
\hline
\end{tabular}

Igualmente significativo es el contraste entre el interés germánico por aunar esfuerzos y la regulación española de un sistema "a dúo", más que dual (Echeverría, 2013b), 
cuando cada vez es más unánime una demanda generalizada de unificar nuestros subsistemas formativos. El RD 1529/2012 contempla por una parte el contrato para la formación y el aprendizaje, prolijamente descrito en los 22 artículos del Título II y, por la otra, la formación profesional del sistema educativo, resumida en los 7 artículos finales, correspondientes al Título III, dividiendo así el perfil único de aprendiz E alumno característico del sistema dual.

El primero - auspiciado por el Ministerio de Empleo (MEYSS) - es para jóvenes que combinan su actividad laboral en una empresa con períodos de formación recibida en centros formativos, relacionados con la actividad que desarrolla en su puesto de trabajo. En él se especifican pormenorizadamente cuestiones laborales como formalización, duración, prórroga y extinción del contrato, período de prueba, jornada laboral, salario, etc.

Esta regulación brilla por su ausencia en la FPD dependiente del Ministerio de Educación (MECD, 2014) y al no existir contrato para realizar la actividad formativa, la formación profesional dual se reduce a proyectos desarrollados dentro de su ámbito (Art. 2.2; 2.3) con la colaboración de las empresas. Esta podrá ser muy fructífera, pero sólo en el mejor de los casos "los alumnos podrán estar becados por las empresas, instituciones, fundaciones, etc., y/o por las Administraciones, en la forma que se determine para cada proyecto" (Art. 33). El alumno seguirá siendo estudiante dentro del entorno laboral, donde completa su formación teórica y se adapta a su realidad, pero de momento se desconoce si la articulación de las anunciadas becas seguirá similar procedimiento al resto de ayudas del MECD o se implantarán mecanismos diferentes.

Si a esto añadimos, que cada una de nuestras 17 Comunidades Autónomas (CCAA), más Ceuta y Melilla, aplica y desarrolla la legislación básica de FP y regula la implementación en su territorio, se puede decir que contamos con 34 modalidades organizativas diferentes de FPD, al depender de organismos disgregados de Educación y de Empleo en cada una de las Comunidades.

A juicio de algunos analistas, "la descentralización de la competencia de educación en las CC.AA. constituye uno de los principales impedimentos para la implementación de la FPD en España" (IESE, 2014, p. 30). Otros incluso consideran que "no hay coordinación de intereses entre las comunidades autónomas y eso dificulta que los conocimientos de los estudiantes sean homologables en todas partes" (Sánchez-Silva, 2015). Hay quien aduce incluso que la simple consulta del Portal Todo FP Formación Profesional Dual del MECD corrobora este hecho y más aún al introducir los términos Formación Profesional Dual en el buscador del MEYSS, que durante un tiempo ha estado remitiendo en primer lugar a la Consejería de Empleo y Seguridad Social en Alemania.

Desde nuestra óptica (Echeverría, 2013b, p. 45), no es problema tanto de descentralización territorial, como de falta de consenso político y administrativo. No ocurre, por ejemplo, en Alemania compuesta por 16 Länder, pero con un sistema de FP dotado de un marco unitario a nivel federal. Como se comentó, los reglamentos de formación y el diseño marco de los planes de enseñanza se elaboran y desarrollan siempre de forma concertada entre el Gobierno Federal, los interlocutores sociales y la Conferencia de Ministros de Educación de los Länder. 


\section{Primeras informaciones}

Transcurrido un trienio desde la promulgación del RD 1529/2012, es exigua la información procedente de los dos Ministerios, que en colaboración las CCAA deben establecer los "mecanismos de recogida y tratamiento de la información obtenida tras el desarrollo e implantación de los proyectos así como los mecanismos de difusión" (Art. 34.2), imprescindibles para el seguimiento y evaluación contemplado en los artículos 16, 24, 25 y 34. Prácticamente se reduce al escueto informe de la Subdirección General de Orientación y Formación Profesional (SGOFP, 2014), donde se refleja:

A) La preponderancia de la formación compartida entre el centro de formación y la empresa (57\%) o con participación de ésta $(28 \%)$ frente al resto de modalidades de desarrollo prescritas en el RD (Art. 3).
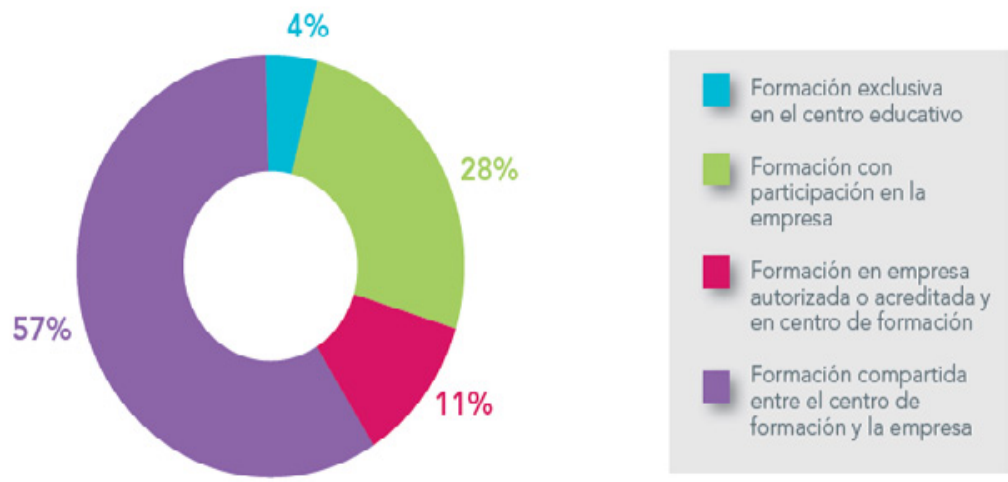

Figura 1. Modalidades de formación. Adaptado de "Seguimiento de Proyectos de Formación Profesional Dual: Curso 2013-2014". En SGOFP, 2014, p. 5)

B) El predominio de ciclos formativos de grado superior (72\%) frente a los de grado medio (28\%), concentrados en las familias profesionales de Fabricación Mecánica (21\%), Electricidad y Electrónica (13\%), Instalación y Mantenimiento (11\%) y Administración y Gestión (10\%), expresados estos últimos valores en porcentaje sobre el total. Por contra, sólo el 1\% del conjunto de proyectos es de la familia Agraria y en 2014 no estaba previsto desarrollo alguno en las familias Marítimo-pesquera, Industrias extractivas, Edificación y obra civil, Vidrio y cerámica, Textil, Confección y piel, y Artesanías.

C) El escaso número de alumnado, centros y empresas implicados, a pesar del notable crecimiento reflejado en la Tabla 2, cuyas cantidades pueden variar ligeramente, al no coincidir las fuentes de información consultadas.

Si se tiene en cuenta, que los alumnos matriculados en FP durante el curso 2014-2015 eran 793.034, los participantes en FPD no llegan al 2\%, porcentaje distante de la tasa de matriculación de este sistema en la UE (14\%) y más aún de la de Alemania (42\%) (MECD, 2014). Aún es más insignificante la proporción de empresas, que según el Directorio Central de Empresas era en España de 3.114.361, a 1 de Enero de 2014 (MIET, 2015). 
Tabla 2

Personas y entidades implicadas en proyectos de FPD

\begin{tabular}{lccc}
\hline \multicolumn{1}{c}{ CURSOS } & $\mathbf{2 0 1 2 - 1 3}$ & $\mathbf{2 0 1 3 - 1 4}$ & $\mathbf{2 0 1 4 - 2 0 1 5}$ \\
\hline Alumnado & 4.292 & 9.555 & 16.200 \\
Centros formativos & 172 & 375 & 720 \\
Empresas & 400 & 800 & 4.878 \\
\hline
\end{tabular}

D) La desigual repartición de centros formativos (Figura 2) y empresas colaboradoras (Figura 3) entre las CCAA, sin disponer análisis contrastados de las causas de tales diferencias, ni siquiera de algunas tan significativas como el crecimiento de empresas participantes en Castilla la Mancha.

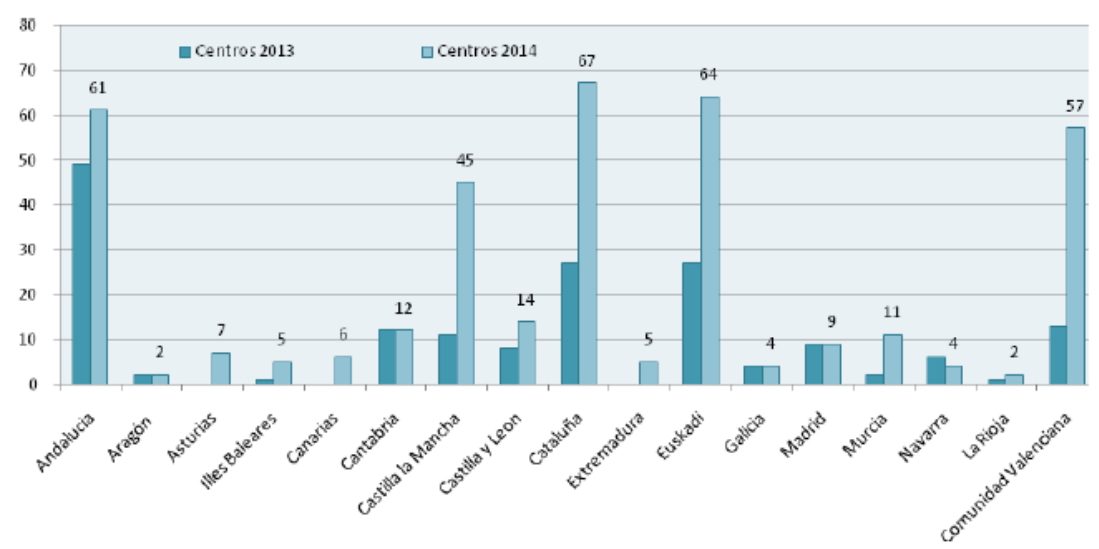

Figura 2. Número de centros con oferta de FPD. Adaptado de "Seguimiento de Proyectos de Formación Profesional Dual: Curso 2013-2014". En SGOFP, 2014, p. 10).

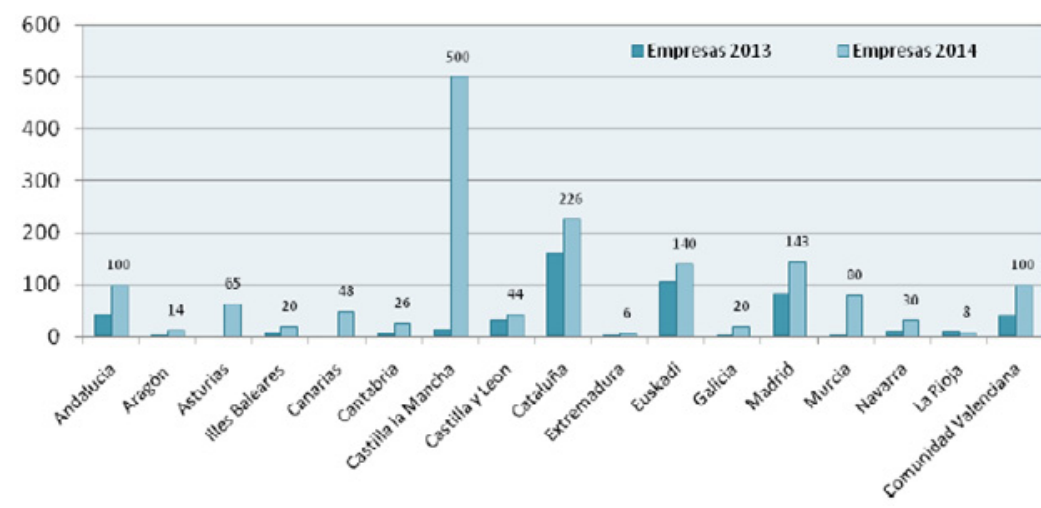

Figura 3. Número de empresas colaboradoras de FPD. Adaptado de "Seguimiento de Proyectos de Formación Profesional Dual: Curso 2013-2014". En SGOFP, 2014, p. 12). 


\section{Investigación-acción}

Una de las pocas investigaciones, que permiten ir más allá de estas cifras, es la de "Evaluación, gobernanza y financiación de los sistemas de Formación Profesional Dual”, dirigida por Felix Rauner del ITB de la Universidad de Bremen, en colaboración con entidades españolas como NOTUS-ASR, bajo la coordinación Oriol Homs. Respaldada por la Alianza Europea de Aprendizaje, se ha realizado en Alemania, España, Italia, Lituania, Portugal y Suecia, financiada por el CEDEFOP (2013a), que publicará próximamente el informe final.

El principal objetivo de este trabajo de 20 meses de duración (finales de 2013 a mediados del 2015) ha sido el de identificar los factores y condiciones que favorecen el establecimiento o desarrollo de la FPD y otras formas similares de aprendizaje, con el fin de proporcionar pautas de actuación concretas y propuestas de cambio en los citados Estados comunitarios, seleccionados entre aquellos con exigua o inexistente implantación de este sistema formativo, frente al prototipo de desarrollo alemán.

Para ello se adoptó un enfoque de investigación-acción con ciertas limitaciones (Rauner et al., 2015), en el que expertos nacionales interesados en FP -representantes de gobiernos, empleadores, formadores,...- evaluamos las estructuras de gobernanza existentes y de escenarios futuros. Concretamente en España llevamos a cabo esta tarea en dos talleres de trabajo (41 participantes en Barcelona, el 26 de junio de 2014 y 49 en Madrid, el 29 de octubre de ese mismo año), donde se consensuó los elementos y criterios del instrumento de evaluación, se razonó las valoraciones personales y se contrastaron diferentes perspectivas.

Se parte de una concepción de la FPD

defined as a formal education and training programme in which learning takes place alternately at a workplace and an educational institution and which leads to an officially recognised vocational qualification. Learning opportunities with these characteristics may also be regarded as 'apprenticeship in the wider sense'. The term 'apprenticeship' (in the narrower sense), on the other hand, applies to programmes in which, on top of the aforementioned characteristics, learners usually have the status of employees and are paid for their work and which normally feature a contractual linkage of the learner with the training enterprise (Rauner et al. 2015, p. 9).

Para analizar su desarrollo, los directores del proyecto presentaron un modelo general de necesidades, criterios de gobernanza y financiación a dos niveles, posteriormente validado: a) Macro: Políticas estratégicas en materia de FP, prioridades de financiación y modelos de organización a nivel nacional; b) Meso: Coordinación y cooperación entre actores - públicos y privados - , agentes sociales, procedimientos de evaluación, estructuras institucionales de formación y autonomía de las instituciones, así como condiciones contractuales de los aprendices.

A su vez, examinaron los acuerdos sistémicos e institucionales, que configuran la implementación de la FPD, desde distintos enfoques conceptuales - Governance Structure (Bertelsman Stiftung, 2009; Rauner, Wittig \& Deitmer, 2010), Feedback Mechanisms 
(CEDEFOP, 2013b) y Skill Formation Regimes (Busemeyer \& Trampusch, 2012; Thelen, 2004) - hasta concluir que el más completo era el de "Skill", que engloba el proceso de desarrollo de capital humano dentro de la economía nacional e integra a los otras dos.

Desde esta perspectiva se acordaron seis criterios normativos con sus elementos constitutivos (Apéndice), que permiten resumir la situación general de un país con respecto a la gobernanza y financiación del aprendizaje en un continuo entre dos límites, que representan las alternativas extremas teóricamente posibles para cada criterio, mediante el ecualizador de Schimank (2007) (Figura 4).

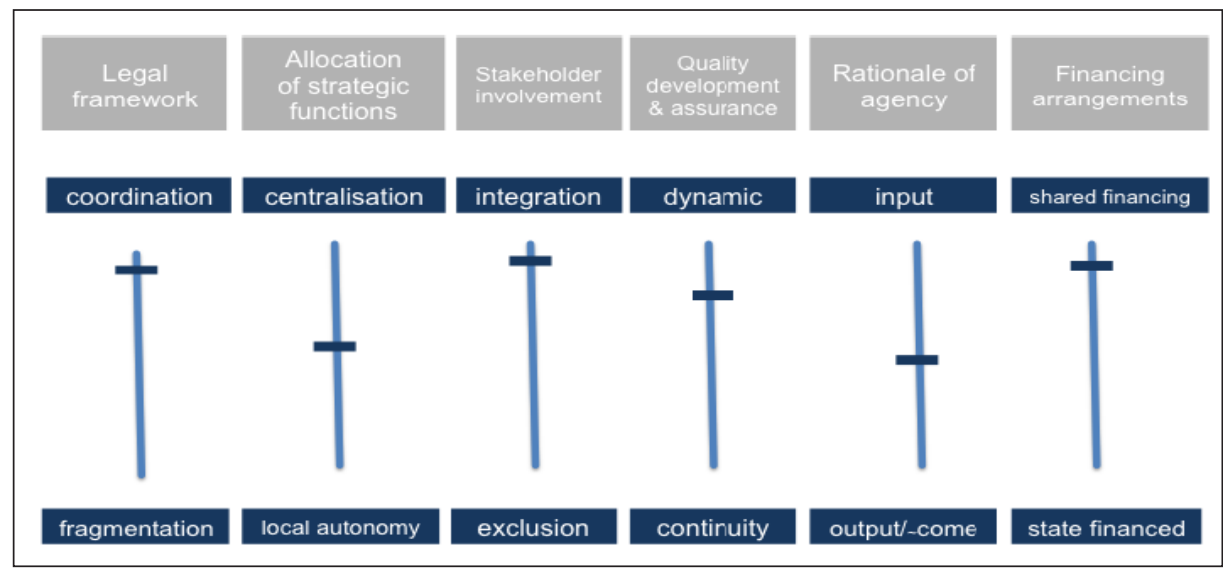

Figura 4. Ecualizador de la gobernanza y financiación del aprendizaje con la configuración "ideal" de los 6 criterios normativos principales. Adaptado de "Die Governance_Perspektive:

Analytisches Potenzial und anstehende konzeptionelle Fragen" por U. Schimank, 2007, Educational Governance: Handlungskoordination und Steuerung im Bildungssystem, p. 240)

La pestaña de la barra de control se desplaza entre los dos extremos de los seis criterios indicando que lo ideal es en el: $1^{\circ}$ ) Alto nivel de coordinación; $2^{\circ}$ ) Distribución equilibrada de funciones entre los niveles nacional y local; $3^{\circ}$ ) Alto grado de participación, integración y coordinación de órganos e interlocutores implicados; 4ำ) Dinamismo en el proceso de innovación continua sin excesivas prisas; $5^{\circ}$ ) Equilibrio entre criterios de acceso y orientación de resultados; $6^{\circ}$ ) Importantes acuerdos de financiación, costos $\mathrm{y}$ beneficios entre todas las partes interesadas.

\section{Valoración de expertos}

En comparación con este marco normativo, los promedios de valoraciones de los expertos españoles, contrastadas en los talleres de Barcelona y Madrid, se distribuyen como se muestra en la Figura 5, con un criterio más que en el anterior, al haberse deslindado en España la orientación hacia los resultados (5) de la de entrada (6).

Las diferencias entre puntuaciones máximas y mínimas evidencian notables desacuerdos, debidos probablemente a la falta de consenso en la misma concepción del sistema (CE, 2012b). Algunos expertos respondieron a las cuestiones planteadas pen- 
sando en el modelo de dualidad integrada, a semejanza del sistema alemán que preconiza el RD 1529/2012. Otros, sin embargo, las juzgaban conforme al modelo de dualidad alterna de la FP regulada por los RD 1538/2006 (Art.11) o por el 1147/2011 (Art.25) que contempla el módulo de Formación en el Centros de Trabajo en todos los ciclos de grado medio y superior.

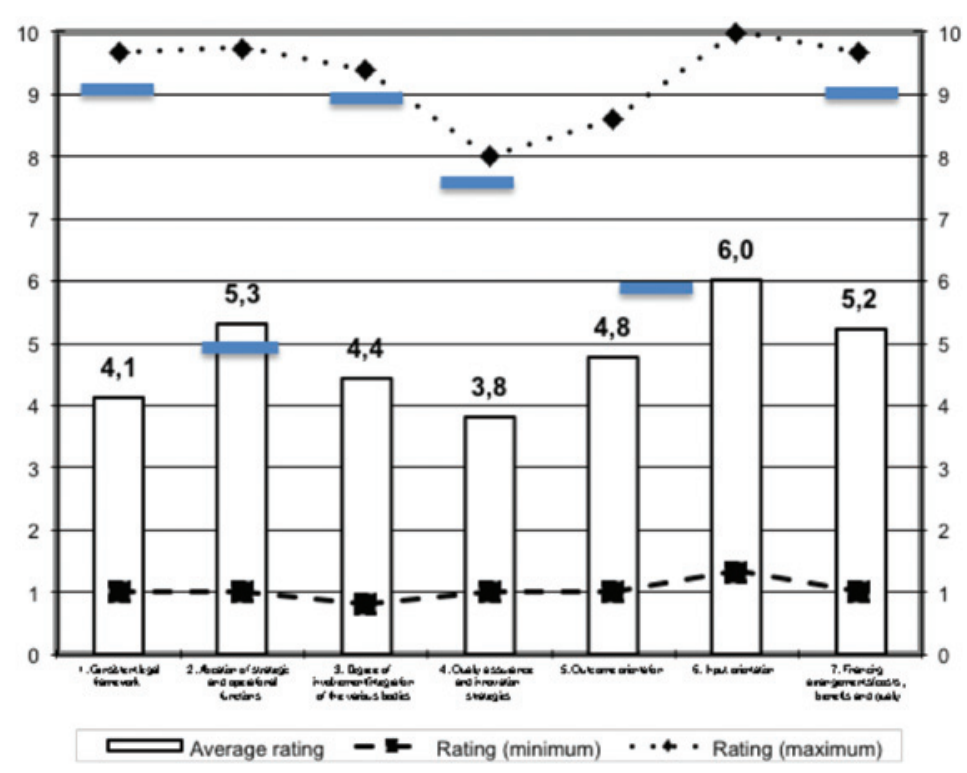

Figura 5. Ecualizador de la gobernanza y financiación del aprendizaje con la configuración "ideal" de los 6 criterios normativos. Adaptado de "Governance and financing of apprenticeship" por Rauner, F. et al. (Dir.) 2015, p. 210.

\section{Coherencia del marco legal}

Esta diferencia conceptual se refleja especialmente en este primer criterio, al carecer España de un marco normativo único y coherente para el "modelo integrado dualidad" regulado en RD 1529/2012. Si bien es un decreto de ámbito nacional, no establece un único sistema de FPD, lo cual está propiciando que las diferentes CCAA desarrollen distintos modelos con diferentes regulaciones.

La mayoría de los expertos coinciden en que el proceso emergente carece de coordinación a nivel nacional, resaltando algunos que el gobierno nacional no ha establecido convenientemente un conjunto de procedimientos destinados a regular la relación entre centros formativos / alumnado / empresas. Otros, sin embargo, juzgan beneficiosa esta indefinición, en cuanto favorece la libertad de las CCAA, para experimentar diferentes estilos de gobernanza y mecanismos de regulación.

El hecho es que las normas reguladoras de la FPD en España pertenecen a distintos ámbitos jurídicos y existen diversos departamentos responsabilizados del establecimiento de las políticas formativas. Se precisa que los actores y las instituciones 
encargadas de aplicarlas acuerden más y mejor los objetivos principales, compartan la estrategia a seguir, se coordinen eficazmente mediante cauces de comunicación funcionales.

\section{Asignación de funciones estratégicas y operativas}

Como se plasma en los gráficos anteriores, el principio de subsidiaridad, por el cual se asignan responsabilidades a diferentes niveles, se respeta legalmente y en la práctica. A juicios de los expertos, existe un reparto equilibrado de funciones entre el MECD, las autoridades educativas de las CCAA y los centros formativos.

Los perfiles ocupacionales y los planes de estudio se definen a nivel nacional, mientras que el desarrollo de los planes formativos corresponde a los actores a nivel local. Los centros de aprendizaje gozan de suficiente autonomía para adaptar los contenidos formativos a sus específicos contextos locales.

Algunos expertos incluso se refieren a ejemplos concretos de empresas que han creado centros de formación adaptados a sus necesidades que ofrecen titulaciones oficiales de FP. Sin embargo, otros plantean la dificultad de conseguir que los contenidos formativos sean relativamente similares, independientemente de la empresa en que el alumnado desarrolle el aprendizaje, sobre todo por el tamaño reducido de las empresas españolas.

\section{Grado de Participación / Integración de los distintos Órganos}

La mayoría de los expertos consideran que la normativa vigente no define adecuadamente las responsabilidades de todos los organismos implicados en la implantación y desarrollo de la FPD. Tampoco garantiza que los actores y socios puedan participar en el diálogo requerido a nivel nacional, regional y local, de acuerdo a sus competencias e intereses; Especialmente entre expertos de los interlocutores sociales, centros de formación y equipos de investigación sobre FP.

Algunos echan a faltar alguna institución que potencie, modere y coordine las relaciones entre estas entidades y la participación adecuada de todas ellas (diálogo), cuya existencia mejoraría la calidad de la cooperación de todos los órganos involucrados en la organización y conformación de la FPD.

Esta deficiencia aún es más significativa, al contrastarla con la metodología del sistema tradicional de FP, donde Instituto Nacional de Cualificaciones Profesionales (INCUAL) dirige 26 grupos - uno por cada familia profesional- de expertos de ámbito laboral y del de formación, que participan en el diseño de las cualificaciones profesionales o perfiles ocupacionales.

\section{Garantía de calidad y estrategias de innovación}

Este es el criterio peor valorado de los seis contemplados, al considerar los expertos que el marco legal no impulsa explícitamente la innovación. Según ellos, tampoco aborda convenientemente el seguimiento y control de la implantación y desarrollo de la FPD, la valoración de los planes formativos, ni los métodos e instrumentos de 
evaluación de los procesos de aprendizaje. No es de extrañar, por tanto, que reclamen mayor conocimiento de cuestiones claves como la formación en el lugar de trabajo, los itinerarios profesionales del alumnado, los índices de inserción laboral, las tasas de deserción, etc.

En general, brilla por su ausencia la más mínima alusión a la investigación como estrategia de innovación, que permita analizar tendencias nacionales e internacionales de FP, fundamentarla psicopedagógicamente o esclarecer objetivos, contenidos y procedimientos, entre otras funciones.

Para la mayoría de los expertos es especialmente significativa la nula referencia al régimen de capacitación y cualificación de sus principales agentes, no solo para acceder a los puestos de trabajo, sino también para el desarrollo profesional continuo, tanto de los profesores de los centros de FP, como de los instructores de las empresas.

\section{Equilibrio entre la orientación de entrada y a resultados}

Los expertos coinciden en valorar bastante positivamente los criterios de acceso, al permitir el marco legal cierto margen de maniobra para que los agentes del sistema puedan conformar los procesos formativos conforme a las necesidades locales.

No es tan bien valorada la orientación a resultados, si bien es bastante acorde al criterio normativo. Por una parte, se espera que al igual que en el sistema tradicional de FP se continúen evaluando los logros alcanzados mediante los habituales indicadores objetivos. Sin embargo, no está tan claro si la mayoría de los exámenes están orientados a los resultados de aprendizaje en términos de competencias o si permiten el reconocimiento de trayectorias individuales de aprendizaje o si el desarrollo profesional de quienes completen la FPD se va a tener en cuenta para adaptar los perfiles a las demandas del mercado laboral y a las necesidades de desarrollo de las trayectorias individuales.

\section{Acuerdos de financiación / costos, beneficios y calidad}

El mismo hecho de que no dispongamos de un sistema nacional de FPD, propiamente dicho, puede explicar la falta de acuerdo entre los expertos sobre este criterio de la financiación.

Algunos juzgan que el Estado es el principal responsable de la misma, al igual que en la FP tradicional, si bien las empresas deberían asumir algunos costos mediante contratos de trabajos. Otros argumentan que el alumnado recibe pequeños ingresos en algunas CCAA, asumiendo por tanto parte de los gastos. Cada una de ellas afronta la financiación del sistema a su forma y manera, al no estar claro el modelo a seguir, ya que la normativa nacional no prescribe convenientemente la relación entre el alumnado y la empresa.

En términos generales, los expertos reconocen que estamos avanzando en la valoración social de la FP en general y del sistema dual en particular, pero es preciso superar obstáculos como los de presupuestos restrictivos, pequeño tamaño de las empresas colaboradoras, bajo nivel de confianza entre interlocutores sociales y el gobierno, inconsistencia del marco legal y gran diversidad territorial. 


\section{Conclusiones y recomendaciones}

Ante esta situación, es imprescindible potenciar al máximo el desarrollo de investigaciones evaluativas sobre el cúmulo de experiencias implementadas a lo largo y ancho del territorio nacional. Es a partir de estos resultados, como es más correcto proceder a la revisión de la regulación actual, hasta alcanzar acuerdos globales sobre las reformas a introducir y los objetivos a lograr en la próxima legislatura, contando con la participación de los principales actores - estado, CCAA, agentes económicos y sociales -.

Algunas de estas reformas del RD 1529/2012 se vienen solicitando desde la misma publicación en el BOE, como la necesidad de: a) Clarificar el concepto de FPD, a la luz de requerimientos como los del Comunicado de Brujas, de la Alianza Europea de Aprendizaje, CEDEFOP, etc.; b) Relacionar mejor el contrato con los programas de formación en aprendizaje, para asegurar la consecución de las titulaciones y de la cualificación correspondiente; c) Regular a través de convenios colectivos sectoriales las condiciones de trabajo del periodo de estancia formativa en la empresa; d) Reglamentar la formación de los tutores de empresa, etc.

Pero después de tres años de experimentación, lo esencial sigue siendo lograr un marco jurídico coherente, aprobado a nivel nacional y coexistente con las estructuras de gobierno autónomas, que permita actuar con la mayor flexibilidad posible en un entorno "glocalizado", donde cada vez es más necesario pensar globalmente y actuar localmente.

Dentro de este marco y con este espíritu, es prioritario: a) Conseguir un sistema formativo de calidad y equitativo, que homogeneíce la relación de las empresas con el alumnado en todo el Estado, independientemente de su tipo y tamaño; b) Determinar adecuadamente la financiación del sistema con las contribuciones de las CCAA y empresas al aporte fundamental del Estado; c) Estimular la participación de las empresas, especialmente la de las Pymes, estableciendo incentivos fiscales en el impuesto de sociedades, que permitan desgravar sus inversiones en formación; d) Lograr la mayor implicación posible de los interlocutores sociales tanto en el diseño como en el desarrollo del sistema, donde la negociación colectiva sectorial debería desempeñar un papel destacado.

Ahora bien, de poco servirán todas estas medidas, sin un amplio conocimiento y posterior respaldo de la FP en general y de la Dual en particular, en una sociedad de hidalgos como la española, partidaria del "white collar" y bastante desorientada sobre la configuración de la profesionalidad. Para ello, es preciso potenciar al máximo el esclarecimiento de posibilidades con futuro, mediante la elección de alternativas acordes al potencial y proyecto vital de cada persona y contrastadas con las ofertadas por el entorno.

Concretamente necesitamos el desarrollo de procesos de orientación profesional coordinada (Echeverría \& Martínez Muñoz, 2014), como viene reclamando el Consejo y los Representantes de los Gobiernos de los Estados de la UE desde la Resolución de 2008, que en su principio cuarto insta: a) Desarrollar una cultura común -particularmente de garantía de calidad- a los distintos servicios competentes a nivel local, regional y nacional; b) Fomentar mecanismos de coordinación y cooperación; 
c) Introducir un elemento de orientación en las estrategias nacionales de educación y formación, así como en las del mercado de trabajo, según la configuración elegida por cada Estado miembro.

En síntesis, apremia sentar las bases de un sistema integrado de información y orientación (Echeverría \& Martínez Clares, 2015) accesible, cercano a todos y con garantía de calidad, que asegure su control y mejora permanente. Un sistema en el que sus agentes y los distintos tipos de servicios compartan una misma cultura de la orientación, donde se coordinen planes de acción funcionales, eficientes y eficaces, desarrollados por profesionales con adecuada formación inicial, continua y específica para los servicios que prestan.

\section{Referencias}

Alianza para la Formación Profesional Dual [AFPD]. (2015). Alianza para la Formación Profesional Dual. Barcelona, España: Fundación Bertelsmann. Recuperado de http:/ www.alianzafpdual.es/sobre-nosotros/alianza-fp-dual/

Bankia (2015). Centro Dual + para la Investigación de la FP Dual. Madrid, España: Bankia. Recuperado de http://fpdualbankia.es/centro-dual/index.html

Bertelsmann Stiftung (Ed.). (2009). Steuerung der beruflichen Bildung in internationalen Vergleich. Gütersloh, Alemania: Verlag Bertelsmann Stiftung. Recuperado de https:// www.bertelsmann-stiftung.de/de/publikationen/publikation/did/steuerung-derberuflichen-bildung-im-internationalen-vergleich/

Bundesministerium für Bildung und Forschun [BMBF]. (2012). Berufsbildungsbericht 2012. Berlin: Autor. Recuperado de https://www.bmbf.de/pub/bbb_2012.pdf

Busemeyer, M. R. \& Trampusch, C. (2012). The Comparative Political Economy of Collective Skill Formation. En M. R. Busemeyer \& C. Trampusch (Eds.), The Political Economy of Collective Skill Formation (pp. 3-38). Oxford: Oxford University Press.

BUSINESSEUROPE (2012). Creating opportunities four youth. How to improve the quality and image of apprenticeships. Brussels: Social Affairs Department. Recuperado de https://www.businesseurope.eu/publications/creating-opportunities-youth-howimprove-quality-and-image-apprenticeships

Centro Europeo para el Desarrollo de la Orientación Profesional [CEDEFOP]. (2013a). Anual List of Contracts: 2013-0132 Governance and Financing of Apprenticeship. Thessaloniki: Publications Office. Recuperado de http://docz.io/doc/1725444/annual-listof-contracts---2013

Centro Europeo para el Desarrollo de la Orientación Profesional [CEDEFOP]. (2013b). Renewing VET provision: Understanding feedback mechanisms between initial VET and the labour market. Luxembourg: Publications Office. (Cedefop Research paper 37). doi: $10.2801 / 32921$.

Comisión Europea [CE]. (2008). Incluir mejor la orientación permanente en las estrategias permanentes de educación y formación permanente. Resolución del Consejo y de los Representantes de los Gobiernos de los Estados (2008/C 319/02). Recuperado de http://eur-lex.europa.eu/legal-content/ES/TXT/PDF/?uri=CELEX:42008X1213(02)\& from $=\mathrm{ES}$ 
Comisión Europea [CE]. (2010). Comunicado de Brujas sobre una cooperación europea reforzada en materia de educación y formación profesionales para el período 2011-2020. Recuperado de http://ec.europa.eu/education/policy/vocational-policy/doc/brugescom_es.pdf

Comisión Europea [CE]. (2012a). Un nuevo concepto de educación: invertir en las competencias para lograr mejores resultados socioeconómicos. Bruselas: COM (2012) 669 final. Recuperado de http://eur-lex.europa.eu/resource.html?uri=cellar:5e4773cc-80ba45df-a2c9-9b70e9cf7dd4.0001.03/DOC_1\&format=PDF

Comisión Europea [CE]. (2012b). Desempleo juvenil: dos estudios señalan la utilidad del aprendizaje y las prácticas profesionales, así como que deben mejorarse. Bruselas: (IP/12/731). Recuperado de http://europa.eu/rapid/press-release_IP-12-731_es.htm

Comisión Europea [CE]. (2013). European Alliance for Apprenticeships. Council Declaration. Brussels: 14986/13. Recuperado de http://www.consilium.europa.eu/uedocs/ cms_data/docs/pressdata/en/lsa/139011.pdf

European Alliance for Apprenticesships [EAA]. (2013). Declaration of the European Social Partners, the European Commission and the Lithuanian Presidency of the Council of the European Union. Lithuania: Autor. Recuperado de http://ec.europa.eu/social/ BlobServlet?docId=14331

Echeverría, B. (2013a). Aprendizajes profesionales en España. En Colectivo de Investigación en Colectivos Ocupacionales [CIFO]. (Eds.). Formación para el trabajo en tiempos de crisis: Balance y prospectiva (pp. 37-48). doi: 10.13140/2.1.3376.4806

Echeverría, B. (2013b). Formación Profesional ¿a dúo o dual?. Educaweb. Recuperado de http://www.educaweb.com/noticia/2013/02/25/formacion-profesional-duo-dual-6006/

Echeverría, B. \& Martínez Muñoz, M. [Adapt.]. (2014). Guía de Orientación Profesional Coordinada. Barcelona: Fundación. Bertelsmann. Recuperado de http://www.todofp. es/dctm/todofp/biblioteca/docs-orientacion/guia-orientacion-fundacion-bertelsmann. pdf?documentId=0901e72b81b23587

Echeverría, B \& Martínez Clares, P. (2015). Sistema Integrado de Orientación. Revista Electrónica Interuniversitaria de Formación del Profesorado, 51(18,2). Recuperado de http://www.aufop.com/aufop/uploaded_files/revistas/142928288611.pdf

Hoeckel, K. \& Schwartz, R. (2010). Learning for Jobs. OECD Reviews of Vocational Education and Training. Germany: OECD Publishing. Recuperado de http://www.oecd. org/germany/45668296.pdf

IESE Business School (2014). La Formación Profesional Dual como reto nacional. Una perspectiva desde la empresa. Barcelona: Autor y Citi Foundation. Recuperado de http:// www.iese.edu/research/pdfs/ST-0362.pdf

Ministerios de Educación, Cultura y Deporte [MECD]. (2014). Panorama de la Educación. Indicadores de la OCDE 2014. Madrid: Autor. Recuperado de http://www.mecd.gob. es/dctm/inee/indicadores-educativos/panorama2014/panorama-de-la-educacion2014informe-espanol-05-sep-.pdf?documentId=0901e72b81a722ac

Ministerio de Industria, Energía y Turismo [MIET]. (2015). Retrato de las PYME 2015. Madrid: Autor. Recuperado de http://www.ipyme.org/Publicaciones/Retrato_ PYME_2015.pdf

Organisation for Economic Cooperation and Development [OECD]. (2010). Learning for Jobs. Reviews of Vocational Education and Training. OECD Publishing. Recuperado 
de http://www.oecd.org/edu/skills-beyond-school/oecdreviewsofvocationaleducationandtraining-learningforjobs.htm

Organisation for Economic Cooperation and Development [OECD]. (2011). Preparándose para trabajar. Informe de síntesis de los análisis de la OCDE sobre educación y formación profesional. Barcelona: Fundació Barcelona FP. Recuperado de http://www.redetis. iipe.unesco.org/publicaciones/ocdept.pdf

Rauner, F., Wittig, W. \& Deitmer, L. (2010). Plural Administration in Dual Systems in Selected European Countries. En F. Rauner \& E. Smith (Eds.), Rediscovering Apprenticeship: Re-search Findings of the International Network on Innovative Apprenticeship (Technical and Vocational Education and Training Series, Vol. 11, pp. 31-43). Dordrecht: Springer.

Rauner, F. et al. (Dir.) (2015). Governance and financing of apprenticeship. Contract no 2013-0132/AO/RPA/PLI-ABARA/Apprenticeship/012/13. Final Report. 24 July 2015 (unpublished manuscript).

Real Decreto 1529/2012, de 8 de noviembre, por el que se desarrolla el contrato para la formación y el aprendizaje y se establecen las bases de la formación profesional dual. Boletín Oficial del Estado, núm. 270, de 9 de noviembre de 2012, pp. 78348-78365. Recuperado de https:/www.boe.es/boe/dias/2012/11/09/pdfs/BOE-A-2012-13846.pdf

Sánchez-Silva, C. (2015). El modelo alemán de la FP dual gana alumnos en España. El País Recuperado de http://economia.elpais.com/economia/2015/04/13/actualidad/1428914445_607629. html

Schimank, U. (2007). Die Governance_Perspektive: Analytisches Potenzial und anstehende konzeptionelle Fragen. En H. Altrichter, T. Brüsemeister \& J. Wissinger (Eds). Educational Governance: Handlungskoordination und Steuerung im Bildungssystem (pp. 231-260). Wiesbaden: VS Verlag für Sozialwissenschaften.

Subdirección General de Orientación y Formación Profesional [SGOFP]. (2014). Seguimiento de Proyectos de Formación Profesional Dual: Curso 2013-2014. Madrid: Autor. Recuperado de http://www.mecd.gob.es/prensa-mecd/dms/mecd/prensa-mecd/ actualidad/2014/01/20140113-fpdual-bankia/informe-seguimiento-fpdual.pdf

Thelen, K. (2004). How Institutions Evolve: The Political Economy of Skills in Germany, Brit-ain, the United States and Japan. Cambridge: Cambridge University Press. 


\section{Apéndice}

\section{Evaluación de las estructuras de gobernanza y criterios de financiación en los sistemas de FP Dual}

Nombre del experto:

Los siguientes puntos han de ser puntuados en una escala de 1 (=totalmente falso) a 10 (=totalmente cierto). Se ruega completar cada punto, aunque tenga dudas sobre la puntuación. Se utilizarán las puntuaciones como un punto de partida para el seminario en el cual habrá la oportunidad de discutir y reconsiderar sus puntuaciones.

\begin{tabular}{|c|c|c|}
\hline $\begin{array}{c}\text { Criterio } \\
\text { principal }\end{array}$ & Sub-criterio & $\begin{array}{c}\text { Puntuación del } \\
\text { sub-criterio } \\
(1 \ldots \ldots \ldots 10)\end{array}$ \\
\hline
\end{tabular}

\section{Marco legal coherente}

1.1. Existe un único marco jurídico para la formación de los aprendices y/o para la FP dual.

1.2. Las competencias legislativas están centralizadas /concentradas.

1.3. Existen procedimientos integrados para el desarrollo de los planes de estudios de la formación profesional.

1.4. Hay regulaciones vinculantes sobre la cooperación entre centros de formación de aprendices.

1.5. Los logros obtenidos en ambos centros de formación de aprendices se tienen en cuenta en los planes de estudio.

1.6. El funcionamiento del marco legal es coherente en la práctica.

\section{Asignación de funciones estratégicas y operativas}

2.1. Las disposiciones legales establecen una distinción clara entre la legislación y su aplicación.

2.2. Las responsabilidades se asignan a los diferentes niveles de acuerdo con el principio de subsidiariedad.

2.3. El desarrollo de los perfiles ocupacionales y los planes de estudio se lleva a cabo a nivel nacional, mientras que el desarrollo de los planes de formación se reserva a los actores a nivel local. 

2.4. Los centros de formación de los aprendices tienen autonomía en cuanto a la implementación de la for- mación profesional.

\section{Grado de Participación/Integración de los distintos Órganos}

3.1. Las responsabilidades de los actores están adecuadamente definidas por el marco legal.

3.2. Los interlocutores sociales, los centros de FP y los investigadores participan en el diálogo sobre la FP.

3.3. La coordinación del sistema en su conjunto se asigna a una institución.

3.4. Los procedimientos para el desarrollo de planes de estudios prevén una participación de los interlocutores sociales.

3.5. Hay una cooperación institucionalizada entre los centros de formación de aprendices.

\section{Garantía de calidad y estrategias de innovación}

4.1. Se aplican las condiciones legales para el desarrollo de la formación de los aprendices y/o la FP dual.

4.2. Los planes de estudio son evaluados regularmente.

4.3. La mejora de la cooperación entre los centros de aprendizaje es uno de los tema de los programas de innovación.

4.4. La valoración y la evaluación de las competencias profesionales es uno de los temas de los programas de innovación.

4.5. La investigación EFTP está involucrada en los programas de innovación.

4.6. Existe y se aplica un sistema integral de desarrollo profesional continuo de los profesores y formadores de FP.

\section{5*. Orientación a resultados}

5.1. El marco legal incluye objetivos obligatorios y estándares de comparación para la formación de aprendices y/o la FP dual.

5.2. Se evalúan sistemáticamente los logros de los objetivos generales de la formación de los aprendices y/o de la FP dual. 

5.3. Los resultados de la investigación sobre el de- sarrollo profesional de los graduados son tenidos en cuenta en la toma de decisiones.

5.4. Los exámenes están orientados a los resultados del aprendizaje.

5.5. Los exámenes permiten el reconocimiento de las trayectorias individuales de aprendizaje.

\section{6*. Criterios de acceso}

6.1. El marco jurídico se basa en el principio de los planes de estudio cerrados.

6.2. El margen de maniobra para los actores está estrechamente definido por el marco legal.

6.3. La realización de un plan de estudios específico es un requisito previo para la concesión de la cualificación profesional.

\section{Acuerdos de financiación/costos, beneficios y calidad}

7.1. La formación en la empresa de los aprendices y/o la de FP dual es un sistema autofinanciado.

7.2. Los costes son compartidos entre las partes interesadas (empresarios, estado, alumnos).

7.3. La formación de los aprendices y/o la FP dual es atractiva para todas las partes interesadas: empresas, alumnos y la economía en su conjunto.

Nota*: La versión original aglutina los criterios 5 y 6 en el de Orientación de entrada y de resultados.

Fecha de recepción: 31 de enero de 2016.

Fecha de revisión: 31 de enero de 2016.

Fecha de aceptación: 29 de marzo de 2016. 